\title{
Antibacterial effects of nitric oxide on uropathogenic Escherichia coli during bladder epithelial cell colonization-a comparison with nitrofurantoin
}

\author{
Ravi Vumma ${ }^{1}$, Charlotte Sahlberg Bang ${ }^{1,2}$, Robert Kruse ${ }^{1,2}$, Kjell Johansson ${ }^{1}$ and Katarina Persson ${ }^{1,2}$
}

The Journal of Antibiotics (2016) 69, 183-186; doi:10.1038/ja.2015.112; published online 4 November 2015

\section{INTRODUCTION}

Uropathogenic Escherichia coli (UPEC) is the predominant causative organism of urinary tract infections (UTI) with a high recurrence rate. ${ }^{1}$ Recurrence of UTI may involve intracellular localization of bacterial colonies within the bladder mucosa, a process that could benefit the bacteria in terms of protection against antibiotics and host immune cells., ${ }^{2,3}$ Once internalized, UPEC may multiply and form intracellular bacterial communities with biofilm-like properties ${ }^{4}$ and/ or enter a non-replicating stable and quiescent state that may serve as a source for recurrent UTI. ${ }^{2,5,6}$ A wide range of antimicrobial agents is used for the treatment of UTI but many antibiotics are unable to penetrate biofilm matrix or inhibit bacteria in a metabolically quiescent state. A recent study demonstrated that of seven different functional classes of antibiotics only a few, including nitrofurantoin and some fluoroquinolones, were able to eliminate internalized UPEC within bladder epithelial cells. ${ }^{3}$ Nitric oxide (NO) is a small hydrophobic molecule with antibacterial properties that readily diffuses through lipid bilayer membranes. ${ }^{7}$ During infection various host cells produce NO enzymatically from inducible nitric oxide synthase and NO has a key role in the innate immune response. ${ }^{7}$ It has been shown previously that NO has antibacterial activity against UPEC isolates, including multidrug-resistant extended spectrum beta-lactamaseproducing isolates. ${ }^{8,9}$ Although NO can interact directly with bacteria, it can also be oxidized to reactive nitrogen species. The proposed targets behind the antibacterial effects of $\mathrm{NO}$ and reactive nitrogen species are multiple and involve for example terminal oxidases, hemes and protein thiols. ${ }^{7}$ Thus, as NO easily diffuses across biological membranes and have antimicrobial properties it may be a suitable molecule to target UPEC that are protected within biofilms or host cells. The antibiotic nitrofurantoin has been used for more than 50 years to treat uncomplicated UTI and there has been virtually no acquired resistance to nitrofurantoin, ${ }^{10}$ not even in multidrug-resistant extended spectrum beta-lactamase-producing isolates. ${ }^{11}$ The reason for the retained antimicrobial activity of nitrofurantoin may be due to its multiple sites of attack and multiple mechanisms of action. The mechanism of action of nitrofurantoin is complex and is not completely understood, but nitrofurantoin appears to be enzymatically reduced by nitroreductase to multiple reactive intermediates in the bacterial cell. ${ }^{10}$ The reduced derivatives appear to attack, by nonspecific reactions, bacterial ribosomal proteins and DNA with the final effect being bactericidal. ${ }^{12}$ Reduction of nitrofurantoin generates nitro radical anions in mammalian cells, ${ }^{13}$ but it is unclear if NO is one of the reactive intermediates produced following metabolism of the nitro group by bacterial or mammalian reductases. The aim of the present study was to examine if NO has antibacterial activity on intracellular UPEC and if nitrofurantoin exerts part of its antibacterial activity by release of NO.

\section{MATERIALS AND METHODS}

The UPEC strain NU14, originally isolated from the urine of a cystitis patient, was grown and maintained on tryptic soy agar plates. The human 5637 bladder epithelial cell line (HTB-9, American Type Culture Collection) was grown in 24-well plates until confluence and infected with NU14 in Dulbecco's modified Eagle's medium supplemented with $2 \%$ fetal bovine serum, 2 mM L-glutamine and $1 \mathrm{~mm}$ non-essential amino acids with a multiplicity of infection of 20 bacteria per host cell for $2 \mathrm{~h}$. After $2 \mathrm{~h}$ of infection, the cells were washed with phosphate-buffered saline (PBS) to remove the non-adherent bacteria. A gentamycin protection assay was used to support intracellular growth of UPEC as previously described. ${ }^{3}$ The cells were incubated for 2,4 or $12 \mathrm{~h}$ in fresh Dulbecco's modified Eagle's medium in the absence or presence of the NO-donor 2,2'-(hydroxynitrosohydrazono)bis-ethanimine (DETA)/NO (1 and $4 \mathrm{~mm}$ ) (DETA/NONOate, Alexis Biochemicals, Lausen, Switzerland), nitrofurantoin (30 and $300 \mu \mathrm{g} \mathrm{m}^{-1}$ ) (Sigma-Aldrich, St Louis, MO, USA) or gentamycin $\left(100 \mu \mathrm{g} \mathrm{ml}^{-1}\right)$. The concentrations selected for DETA/NO (1 and $4 \mathrm{~mm}$ ) correspond to the MIC and four times MIC. The concentrations of nitrofurantoin ( 30 and $300 \mu \mathrm{g} \mathrm{ml}^{-1}$ ) were selected based on another study ${ }^{3}$ and represent the range of levels that normally accumulates in human urine during the course of treatment. ${ }^{14}$ Finally, the cells were washed with PBS, lysed with $0.5 \%$ Triton X-100 and bacterial counts (CFU per ml) were determined on tryptic soy agar plates. The host cell viability and adherence were checked throughout the experiments.

${ }^{1}$ School of Health and Medical Sciences, Örebro University, Örebro, Sweden and ${ }^{2}$ RRiSC_Inflammatory Responses and Infection Susceptibility Centre, Örebro University, Örebro, Sweden

Correspondence: Professor K Persson, School of Medicine, Campus USÖ Örebro University, SE-701 82, Örebro, Sweden.

E-mail: katarina.persson@oru.se

Received 12 May 2015; revised 30 September 2015; accepted 8 October 2015; published online 4 November 2015 
Measurement of extracellular NO production was performed using the Griess reaction assay. ${ }^{15} \mathrm{NO}$ is rapidly converted to the more stable end product nitrite and nitrite levels were measured in the cell culture supernatants from infected 5637 bladder cells treated with DETA/NO and nitrofurantoin. Nitrite concentrations were determined by spectrophotometry with a lower detection limit of $1 \mu \mathrm{M}$ nitrite. Detection of intracellular NO was performed by DAF-FMDA and confocal microscopy. Briefly, infected 5637 bladder cells were treated with DETA/NO and nitrofurantoin and incubated with $8 \mu \mathrm{m}$ of the NO indicator DAF-FM-DA (4-amino-5-methylamino-2,7-difluorescein diacetate, Molecular Probes, Eugene, OR, USA) for $30 \mathrm{~min}$. The slides were analyzed using a Leica TCS SP2 confocal laser scanning microscope (Heidelberg GmbH, Wetzlar, Germany) at $488 \mathrm{~nm}$.

\section{RESULTS AND DISCUSSION}

The ability of strain NU14 to colonize 5637 bladder cell was first evaluated. Monolayers of bladder 5637 epithelial cells were infected and incubated with gentamycin to allow establishment of intracellular bacteria. The bacterial counts of NU14 amounted to $4.4 \times 10^{3} \pm 2.3 \times 10^{3} \mathrm{CFU}$ per $\mathrm{ml}(n=3)$ after $16 \mathrm{~h}$ of incubation. A double immunofluorescence staining procedure that can distinguish between adherent extracellular bacteria and intracellular bacteria revealed the presence of both extracellular and intracellular NU14. Thus, in agreement with other studies ${ }^{5,6}$ UPEC was able to colonize and invade bladder epithelial cells by the in vitro colonization method used.

We next studied the effect of the NO-donor DETA/NO and nitrofurantoin on the viability of strain NU14 following colonization of host bladder epithelial cells. The bacterial counts in the absence of any antibiotics (controls) increased $\sim 1000$-fold after $12 \mathrm{~h}$ relative to the numbers of bacteria at $2 \mathrm{~h}$ (Figure 1). DETA/NO at $1 \mathrm{~mm}$ caused a small initial growth inhibition but after $12 \mathrm{~h}$ a partial recovery of growth was found (Figure 1). However, the growth response after $12 \mathrm{~h}$ was significantly $(P<0.01)$ suppressed compared to untreated

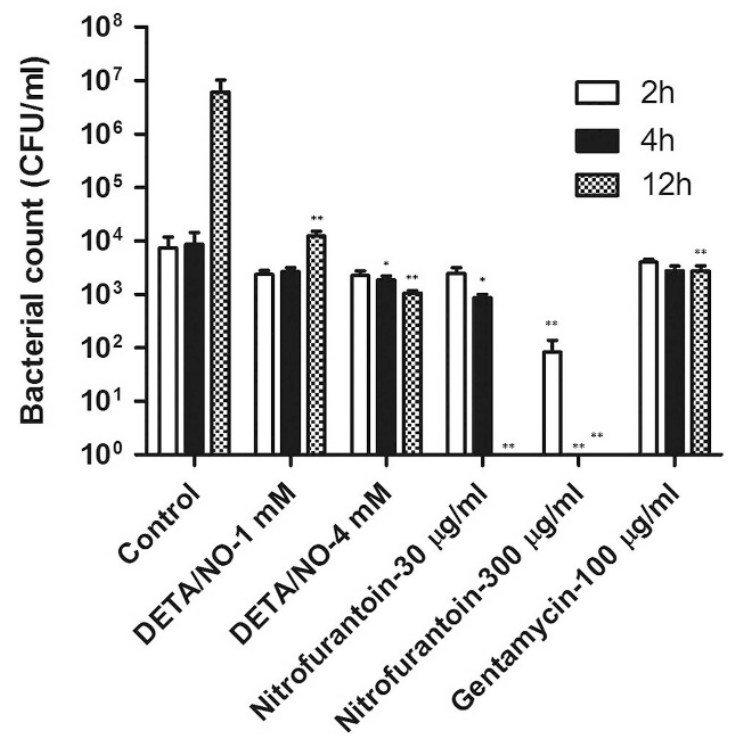

Figure 1 Growth inhibitory effects of DETA/NO and nitrofurantoin on strain NU14 following colonization of bladder epithelial cells. The effect of DETA/ NO ( 1 and $4 \mathrm{~mm}$ ), nitrofurantoin (30 and $300 \mu \mathrm{g} \mathrm{ml}^{-1}$ ) and gentamycin $\left(100 \mu \mathrm{g} \mathrm{ml}^{-1}\right)$ on strain NU14 following colonizing of 5637 bladder epithelial cells after 2, 4 and $12 \mathrm{~h}$ of exposure. Controls were not exposed to antibiotics. Bacterial count is presented as the mean values \pm s.e.m. from three to six independent experiments. Statistical analysis (one-way analysis of variance followed by Bonferroni's test); ${ }^{*} P<0.05$, ${ }^{* *} P<0.01$ versus timematched controls. controls. A higher concentration of DETA/NO (4 mM) caused a prolonged bacteriostatic response with no recovery of growth after $12 \mathrm{~h}$ of incubation (Figure 1). Colonized bacteria can emerge from host cells and subsequently multiply when antibiotics are removed ${ }^{5}$ and resurgence of UPEC from intracellular reservoirs is believed to promote the development of recurrent and chronic UTI. ${ }^{2,6}$ Thus, even if the inhibitory effect of DETA/NO on the number of colonized bacteria was modest, DETA/NO was able to significantly prevent resurgent growth of NU14 when associated with host cells. Nitrofurantoin markedly inhibited the growth of colonized UPEC, as previously reported, ${ }^{3}$ suggesting that nitrofurantoin is able to penetrate the cell membrane of bladder epithelial cells. Nitrofurantoin at 30 $\mu \mathrm{g} \mathrm{ml}^{-1}$ eradicated NU14 after $12 \mathrm{~h}$, whereas a high concentration of nitrofurantoin $\left(300 \mu \mathrm{g} \mathrm{ml}^{-1}\right)$ eradicated NU14 after $4 \mathrm{~h}$ (Figure 1). Visual observation demonstrated that the 5637 bladder epithelial cells remained attached after $12 \mathrm{~h}$ of exposure to DETA/NO and nitrofurantoin. Some bladder cell cytotoxicity was found with the highest concentrations used as determined by lactate dehydrogenase release, but the antibacterial effects of DETA/NO and nitrofurantoin did not correlate with the cytotoxic effects. A lack of correlation between the antibacterial effect of nitrofurantoin and its cytotoxic effect on 5637 bladder cells was also reported in a study using a similar cell culturebased assay as in our study. ${ }^{3}$ The bacterial counts in cultures treated with gentamycin $\left(100 \mu \mathrm{g} \mathrm{ml}^{-1}\right)$, an antibiotic with low host cell membrane permeability, did not decrease during the $12 \mathrm{~h}$ exposure time (Figure 1). These data show that DETA/NO and, in particular, nitrofurantoin have growth inhibitory effects on colonized UPEC.

In $E$. coli, the nitro group of nitrofurantoin is turned into antimicrobial active compounds by nitroreductases encoded by $n s f$ genes. ${ }^{16}$ Although the toxic products formed following reduction of nitrofurantoin remains unclear, intermediate nitroso compounds and formation of hydroxylamine products have been proposed. ${ }^{12}$ We next examined if the antibacterial mechanisms of nitrofurantoin may involve release of NO. The structures of nitrofurantoin and DETA/ $\mathrm{NO}$ are shown in Figure 2a. NO is rapidly converted to the more stable end product nitrite ${ }^{15}$ and a nitrite assay was used to examine whether nitrofurantoin releases NO into the cell culture medium from monolayers of 5637 cells colonized by NU14. However, nitrite was not detectable in medium from cultures exposed to nitrofurantoin (300 $\mu \mathrm{g} \mathrm{ml}^{-1}$ ) (Figure 2b). DETA/NO is a NO-donor that decomposes spontaneously ${ }^{17}$ and our data confirm that high levels of NO (measured as nitrite) are produced in the extracellular milieu within $2 \mathrm{~h}$ and that NO levels are sustained during the $12 \mathrm{~h}$ study period (Figure 2b). To examine whether NO could be detected intracellular in bladder 5637 cells exposed to DETA/NO and nitrofurantoin, a fluorescent indicator of intracellular NO production in living cells was used. ${ }^{18}$ The NO-indicator DAF-FM-DA is converted to DAF-FM by intracellular esterases and upon reaction with NO fluorescence is formed. The cytoplasm of DETA/NO-exposed bladder cells exhibited a fluorescence signal with heterogeneous distribution where some cells showed an intense signal and some a weaker signal (Figure 2c). In some cells a punctuated pattern of fluorescence was observed. Noteworthy, the antibacterial effect of DETA/NO against UPEC is more pronounced in experiments without host cells ${ }^{9}$ than in experiments with colonized UPEC. This is likely due to the biological reactivity of the free radical $\mathrm{NO}$ with host cell targets that may result in lower antibacterial concentration of NO in the intracellular milieu. However, importantly our data demonstrate that DETA/NO-derived NO is able to reach the cytosol of host bladder cells to target intracellular UPEC. Treatment of cells with nitrofurantoin did not result in any distinct or consistent fluorescence signal inside the cells, 
a

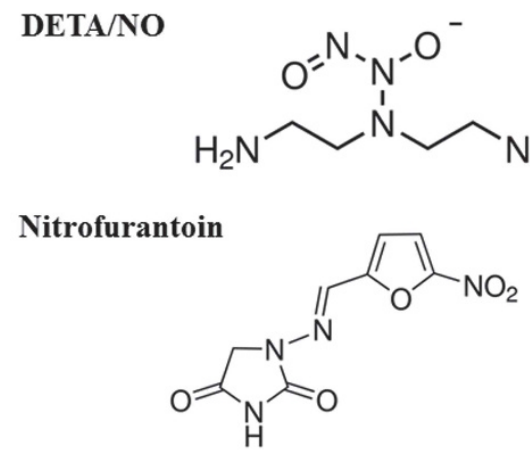

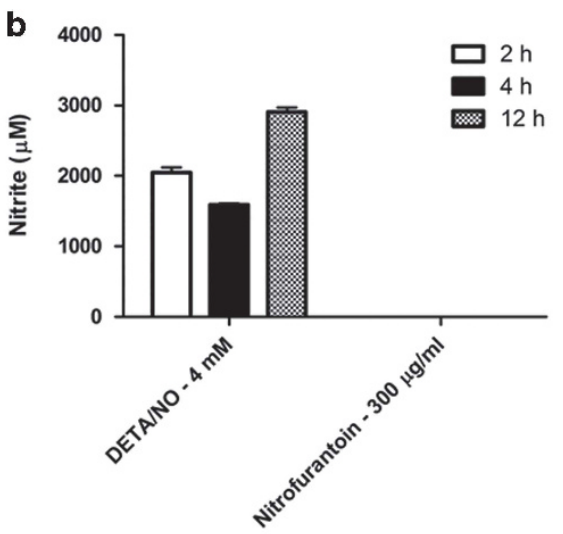
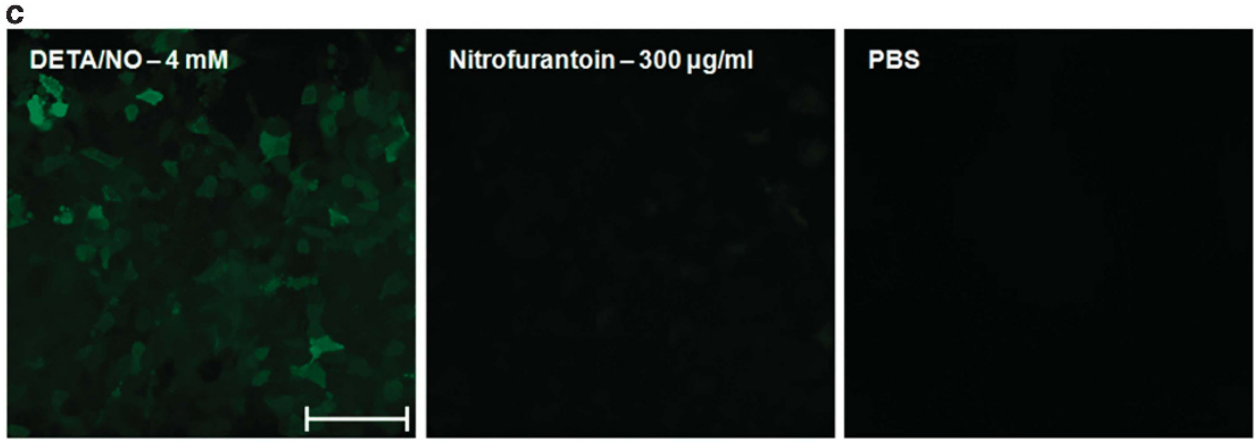

Figure 2 A comparison of extracellular and intracellular production of NO by DETA/NO and nitrofurantoin. (a) Structure of DETA/NO and nitrofurantoin (b) Extracellular nitrite production from 5637 bladder epithelial cells colonized by NU14 and exposed to DETA/NO ( $4 \mathrm{mm)}$ and nitrofurantoin ( $300 \mu \mathrm{g} \mathrm{ml}^{-1}$ ) for 2, 4 and $12 \mathrm{~h}$. Data are expressed as mean \pm s.e.m. from three independent experiments. (c) Images from confocal microscopy showing 5637 bladder epithelial cells colonized by NU14 and exposed to DETA/NO ( $4 \mathrm{~mm})$, nitrofuratoin $\left(300 \mu \mathrm{g} \mathrm{ml}{ }^{-1}\right)$ or PBS on reaction with the NO-indicator DAF-FM-DA ( $\left.8 \mu \mathrm{m}\right)$. Scale bar $=75 \mu \mathrm{m}$

although a weak background staining was noted (Figure 2c). Thus, neither nitrite nor NO was detected in infected 5637 bladder cells exposed to nitrofurantoin by the Griess assay or the fluorescent NOindicator DAF-FM-DA. This suggest that NO is not formed as a byproduct from microbial or mammalian nitroreductase metabolism or from nitroreductase-independent activation of nitrofurantoin. However, it is likely that detection of small amounts of $\mathrm{NO}$ would have required more sensitive methods. Moreover, production of some antimicrobial nitrogen species (for example, $\mathrm{N}_{2} \mathrm{O}_{3}, \mathrm{~N}_{2} \mathrm{O}_{4}, \mathrm{HNO}_{2}$, $\mathrm{ONOO}^{-}$and S-nitrosothiols) may have occurred without detectable $\mathrm{NO}$ or nitrite formation.

In contrast to the bactericidal properties of nitrofurantoin, the antibacterial effects of $\mathrm{NO}$ was bacteriostatic. ${ }^{9}$ Many bacteria have evolved mechanisms of protection from NO including activation of flavohemoglobin, an enzyme with NO dioxygenase activity, that detoxifies NO to nitrate. ${ }^{19}$ We have recently shown that the bacteriostatic effects of DETA/NO against multidrug-resistant UPEC can be substantially prolonged by pharmacological inhibition of flavohemoglobin. ${ }^{9}$ Identifying new treatment options with improved effectiveness against intracellular localized UPEC or within biofilms is essential. Several NO-based technologies have been developed to control bacteria for example, NO-releasing materials and surface coatings for the prevention and dispersal of biofilms, including NOimpregnated Foley urinary catheters. ${ }^{20}$ In the present study, we expand this knowledge and suggest that NO has a potential to target UPEC also when associated with host bladder epithelial cells. Nitrofurantoin was effective against colonized UPEC but nitrofurantoin metabolism does not appear to involve release of NO.

\section{ACKNOWLEDGEMENTS}

We acknowledge support from the Swedish Council for Working Life and Social Research (FAS), Nyckelfonden at Örebro University Hospital and the Faculty of Medicine and Health at Örebro University.

1 Ronald, A. The etiology of urinary tract infection: traditional and emerging pathogens. Dis. Mon. 49, 71-82 (2003).

2 Dhakal, B. K., Kulesus, R. R. \& Mulvey, M. A. Mechanisms and consequences of bladder cell invasion by uropathogenic Escherichia coli. Eur. J. Clin. Invest. 38 (Suppl 2), 2-11 (2008).

3 Blango, M. G. \& Mulvey, M. A. Persistence of uropathogenic Escherichia coli in the face of multiple antibiotics. Antimicrob. Agents Chemother. 54, 1855-1863 (2010).

4 Anderson, G. G. et al. Intracellular bacterial biofilm-like pods in urinary tract infections. Science 301, 105-107 (2003).

5 Mulvey, M. A., Schilling, J. D. \& Hultgren, S. J. Establishment of a persistent Escherichia coli reservoir during the acute phase of a bladder infection. Infect. Immun. 69, 4572-4579 (2001).

6 Eto, D. S., Sundsbak, J. L. \& Mulvey, M. A. Actin-gated intracellular growth and resurgence of uropathogenic Escherichia coli. Cell. Microbiol. 8, 704-717 (2006).

7 Fang, F. C. Antimicrobial reactive oxygen and nitrogen species: concepts and controversies. Nat. Rev. Microbiol. 2, 820-832 (2004).

8 Carlsson, S., Weitzberg, E., Wiklund, P. \& Lundberg, J. O. Intravesical nitric oxide delivery for prevention of catheter-associated urinary tract infections. Antimicrob. Agents Chemother. 49, 2352-2355 (2005).

9 Bang, C. S. et al. The antibacterial effect of nitric oxide against ESBL-producing uropathogenic $\mathrm{E}$. coli is improved by combination with miconazole and polymyxin $\mathrm{B}$ nonapeptide. BMC Microbiol. 14, 65 (2014).

10 McOsker, C. C. \& Fitzpatrick, P. M. Nitrofurantoin: mechanism of action and implications for resistance development in common uropathogens. J. Antimicrob. Chemother. 33(Suppl A), 23-30 (1994).

11 Sanchez, G. V., Baird, A. M., Karlowsky, J. A., Master, R. N. \& Bordon, J. M. Nitrofurantoin retains antimicrobial activity against multidrug-resistant urinary Escherichia coli from US outpatients. J. Antimicrob. Chemother. 69, 3259-3262 (2014). 
12 Lu, C., McCalla, D. R. \& Bryant, D. W. Action of nitrofurans on E. coli: mutation and induction and repair of daughter-strand gaps in DNA. Mutat. Res. 67, 133-144 (1979).

13 Rao, D. N. \& Mason, R. P. Generation of nitro radical anions of some 5-nitrofurans, 2- and 5-nitroimidazoles by norepinephrine, dopamine, and serotonin. A possible mechanism for neurotoxicity caused by nitroheterocyclic drugs. J Biol. Chem. 262, 11731-11736 (1987).

14 Lorian, V. Antibiotics in laboratory medicine. 5th edn. (Lippincott Williams \& Wilkins, Philadelphia, 2005).

15 Granger, D. L., Taintor, R. R., Boockvar, K. S. \& Hibbs, J. B. Jr. Measurement of nitrate and nitrite in biological samples using nitrate reductase and Griess reaction. Methods Enzymol. 268, 142-151 (1996).
16 Bryant, D. W., McCalla, D. R., Leeksma, M. \& Laneuville, P. Type I nitroreductases of Escherichia coli. Can. J.Microbiol. 27, 81-86 (1981)

17 Keefer, L. K., Nims, R. W., Davies, K. M. \& Wink, D. A. "NONOates" (1-substituted diazen-1-ium-1,2-diolates) as nitric oxide donors: convenient nitric oxide dosage forms. Methods Enzymol. 268, 281-293 (1996).

18 Lepiller, S. et al. Imaging of nitric oxide in a living vertebrate using a diaminofluorescein probe. Free Radic. Biol. Med. 43, 619-627 (2007).

19 Poole, R. K. \& Hughes, M. N. New functions for the ancient globin family: bacterial responses to nitric oxide and nitrosative stress. Mol. Microbiol. 36, 775-783 (2000).

20 Regev-Shoshani, G., Ko, M., Miller, C. \& Av-Gay, Y. Slow release of nitric oxide from charged catheters and its effect on biofilm formation by Escherichia coli. Antimicrob. Agents Chemother. 54, 273-279 (2010). 